\title{
BMJ Open Exploring patients' understanding of antibiotic resistance and how this may influence attitudes towards antibiotic use for acute respiratory infections: a qualitative study in Australian general practice
}

Mina Bakhit, ${ }^{\circledR}$ Chris Del Mar, Elizabeth Gibson, Tammy Hoffmann

To cite: Bakhit M, Del Mar C, Gibson $\mathrm{E}$, et al. Exploring patients' understanding of antibiotic resistance and how this may influence attitudes towards antibiotic use for acute respiratory infections: a qualitative study in Australian general practice. BMJ Open 2019;9:e026735. doi:10.1136/ bmjopen-2018-026735

- Prepublication history for this paper is available online. To view these files, please visit the journal online (http://dx.doi org/10.1136/bmjopen-2018026735).

Received 17 September 2018 Revised 4 February 2019 Accepted 5 February 2019
D) Check for updates

(c) Author(s) (or their employer(s)) 2019. Re-use permitted under CC BY-NC. No commercial re-use. See rights and permissions. Published by BMJ.

Centre for Research in Evidence-Based Practice, Gold Coast, Queensland, Australia

Correspondence to Professor Tammy Hoffmann; thoffman@bond.edu.au

\section{ABSTRACT}

Objectives To explore patients' or parents of child patients' understanding of antibiotic resistance and aspects of resistance such as resistance reversibility and its spread among those in close proximity, along with how this may influence attitudes towards antibiotic use for acute respiratory infections (ARIs).

Design Qualitative semistructured interview study using convenience sampling and thematic analysis by two researchers independently.

Setting General practices in Gold Coast, Australia. Participants 32 patients or parents of child patients presenting to general practice with an ARI.

Results Five themes emerged: (1) antibiotic use is seen as the main cause of antibiotic resistance, but what it is that becomes resistant is poorly understood; (2) resistance is perceived as a future 'big problem' for the community, with little appreciation of the individual impact of or contribution to it; (3) poor awareness that resistance can spread between family members but concern that it can; (4) low awareness that resistance can decay with time and variable impact of this knowledge on attitudes towards future antibiotic use and (5) antibiotics are perceived as sometimes necessary, with some awareness and consideration of their harms.

Conclusions Patients' or parents of child patients' understanding of antibiotic resistance and aspects of it was poor. Targeting misunderstandings about resistance in public health messages and clinical consultations should be considered as part of a strategy to improve knowledge about it, which may encourage more consideration about antibiotic use for illnesses such as ARIs.

\section{INTRODUCTION}

Antibiotics, which have been critically important for treating infections since their discovery in the 1940s, are accelerating towards weakened effectiveness due to increase in antibiotic resistance. ${ }^{1}$ Antibiotic resistance, which occurs when bacteria change in response to the use of antibiotics
Strengths and limitations of this study

- Thematic analysis was performed by two researchers independently.

- To our knowledge, this is the first study to explore patients' knowledge of the spread of antibiotic resistance between those in close proximity and its decay with time.

- Sample unlikely to be representative of the wider Australian population or illnesses.

and resist the effects of antibiotics, is largely driven by community antibiotic use. ${ }^{2-4}$ Antibiotics are prescribed more in primary care than other health sectors, and often for acute respiratory infections (ARIs), which comprise approximately $10 \%$ of primary care consultations. ${ }^{5}$ Because of high prescribing rates, particularly for common conditions where antibiotics provide little benefit such as sore throat, ${ }^{6}$ acute otitis media (AOM) ${ }^{7}$ and bronchitis, ${ }^{8}$ primary care is targeted for reducing antibiotic prescribing.

Understanding patients' beliefs about antibiotics and reasons for using and not using them can help inform interventions and public campaigns that aim to encourage appropriate antibiotic use. ${ }^{9}$ Research has revealed that patients overestimate the benefits of antibiotics for ARIs, ${ }^{10}$ and their expectations can influence antibiotic prescribing. ${ }^{11}$

Research that has explored the public's understanding of antibiotic resistance, consequences of it, and whether patients consider the threat of resistance when deciding, ideally in conjunction with their clinician, whether to use antibiotics is scarce. ${ }^{912}$ There are also aspects of antibiotic resistance that might affect perceptions about antibiotic 
use, but patients' understanding of and views about these have not been investigated. This includes that antibiotic use increases resistance in the period following use, but this resistance decays with time, ${ }^{4}$ and that resistance can be transmitted between people in close proximity such as family and household members. ${ }^{13}$ How knowledge of this might influence patients' beliefs about antibiotic use for minor self-limiting illnesses such as ARIs is unknown. Such information is needed to ensure that clinical consultations and public health campaigns about antibiotic use cover all the appropriate and relevant key messages.

This study aimed to explore, in a sample of patients, or parents of child patients, presenting to a general practitioner (GP) directly after the decision-making point in a clinical encounter for ARI, their understanding of: (1) antibiotic resistance in general and (2) aspects of antibiotic resistance, including resistance decay and spread among people in close proximity, and how attitudes towards antibiotic use may be influenced by this understanding.

\section{METHODS \\ Design}

This was a qualitative study which used semistructured interviews to explore participants' understanding of antibiotic resistance and implications for decisions about antibiotic use.

\section{Participants and setting}

Recruitment and the interviews occurred in general practices in Southeast Queensland, Australia that had been recruited as part of an ongoing cluster randomised trial. ${ }^{14}$ The trial intervention that was provided to the general practices was three patient decision aids (for AOM), acute sore throat and acute bronchitis) and a $15 \mathrm{~min}$ video that demonstrated shared decision-making. Practices randomised to the control group did not receive any active intervention.

Recruitment of participants for this study occurred between September 2016 and June 2017 from both the intervention and control practices. Practice managers' approvals were obtained through email communication and recruitment days were organised according to each practice's preference. Patients were eligible to participate if they met these criteria. The first was that they were an adult (or parent of a sick child) 18 years or older consulting a consenting GP with one of three ARIs (AOM, acute sore throat, acute bronchitis) for the first time for that illness episode. We recruited adults and children as both experience ARIs and with a few exceptions, the benefits and harms of antibiotics for ARIs, along with the risk and consequences of antibiotic resistance, are similar for both groups. Other criteria were that participants could understand and read English and provide written informed consent.

\section{Patient and public involvement}

No patients or members of the public were involved in the design of this study. However, they were involved in
Box 1 Summary of topic guide for interviews

- 'Usual' approaches of expecting and/or using antibiotics for managing acute respiratory infections, including beliefs about necessity of antibiotics, their benefits and harms and other influences on decision-making about antibiotic use

- Understanding of the meaning of 'antibiotic resistance', its cause/s, and implications of it. (If the participant did not know what resistance was, the interviewer provided a brief explanation before proceeding to next questions).

- Awareness that antibiotic resistance can spread between those in close proximity (such as family and household members) and if unaware, reactions to being told that it can.

- Awareness that antibiotic resistance can decay over time and if unaware, reactions to being told that it can.

the development of the decision aids used by GPs in some of the recruited general practices. Patients were involved in this study as participants. The results of this study were disseminated to interested study participants by email.

\section{Procedure}

The interviews were conducted by one author (MB), using an interview topic guide (summarised in box 1). The topic guide was developed based on a systematic review of relevant literature, ${ }^{12}$ and findings from a cross-sectional study of Australian parents' experiences of ARI management and antibiotic use in primary care..$^{10}$ The questions were piloted with two eligible participants who were not recruited into the study, and minor rephrasing of some questions occurred after piloting.

Some practices organised a room for the interviews, whereas at other practices, the interviews occurred in a private area of the waiting room. The recruitment process differed according to each practice's preference. At some practices, the interviewer (assisted by practice staff) approached only patients who were waiting to see the GPs who were participating. At other practices, the interviewer approached all waiting patients and asked if they were waiting to see one of the participating GPs (GP names were listed and shown to patients). If so, recruitment proceeded. Patient eligibility was determined by asking the patients if they were suffering from one of the following symptoms (sore throat, cough, ear pain), with the diagnosis confirmed afterwards by the treating GP. Potential participants were provided with a verbal explanation of the study and a written study information sheet. After confirming eligibility and obtaining written consent, each participant was interviewed for an average of approximately $15 \mathrm{~min}$ directly after leaving the consultation room. Patients were interviewed directly after the consultation because this is: (1) for most, the time of decision-making about whether to take antibiotics, (2) important for reducing recall bias and (3) enabled face-toface interviews to occur. Interviews were audio recorded, with participants' consent, and transcribed verbatim afterwards. The interview recording was deleted if a patient was diagnosed by their GP as having an illness other than 
an ARI. This occurred for one recording as the patient had a cough from a chronic illness.

\section{Data analysis}

After 26 participants had been interviewed, a preliminary thematic analysis was undertaken. It was decided that data saturation had not occurred, and recruitment of participants continued until data saturation was obtained at 32 participants. This was defined as when no new ideas or constructs emerged from two consecutive interviews. ${ }^{15}$ Two authors (MB and EG) then independently used the process for thematic analysis outlined by Braun and Clarke. ${ }^{16}$ After familiarising themselves with the interview transcripts, they generated overarching themes and subthemes. This was a data-driven process that was partially inductive in nature. The authors compared and discussed their themes and analyses and with the input of an additional researcher (TH), came to consensus. The themes and illustrative quotes were then agreed to by all authors.

\section{RESULTS \\ Participant characteristics}

We approached 208 patients in five general practices: 41 met the inclusion criterion of having an ARI, and of these, 32 (18 adult patients and 14 parents of sick children) consented to participate. The most common reason given for declining participation was insufficient time to be interviewed. Participants' mean age was 38 years (range $18-74)$, the majority were female $(n=25,78 \%)$ and half $(\mathrm{n}=16,50 \%)$ were consulting for an episode of acute bronchitis (table 1).

\section{Themes}

Five themes emerged, and these are presented below and supported by illustrative quotes.

Theme 1: antibiotic use is seen as the main cause of antibiotic resistance, but what it is that becomes resistant is poorly understood

Many participants thought that antibiotic overuse or misuse in people drives antibiotic resistance-'Sometimes people think they need antibiotics. That's where they can lead to resistance because they have them too much' (P03); with a few mentioning other reasons such as antibiotic use in animals; 'Through our food, that sort of thing, it does seem to be a concern now. Like, animals getting fed antibiotics' (P12); or not using the full antibiotic course 'But if you use them ... you don't take the full dose, obviously like in that you've got your certain bugs coming out.' (P25).

Nearly all participants thought that antibiotic resistance is when the body becomes resistant to antibiotics:

'Antibiotic resistance, your body is resistant to it and maybe you've used too much of it... antibiotics' (P16)
'Antibiotic resistance is possibly your body, rejecting the benefits of the antibiotics ... it's almost like the body gets used to the antibiotic' (P10)

'If you take antibiotics too regularly, your body stops, reacting to them, or they stop having an impact' (P04)

Some participants still had misperceptions after the interviewer provided a simple explanation of what antibiotic resistance is ('Antibiotic resistance happens when bacteria change to protect themselves from an antibiotic. They are then no longer killed by that antibiotic'):

'Oh, yeah, see I've never had that sort of problem. I've never heard it. Whenever I've taken it, maybe I wasn't sick enough to sort of resist it. It's always worked. And for the time that I had to take more than once, a repeat, you know.' (P23)

Theme 2: resistance is perceived as a future 'big problem' for the community, with little appreciation of the individual impact of, or contribution to it

Most participants perceived antibiotic resistance as a community problem caused by others who misuse antibiotics:

' $\ldots$ if people are over using it. Yeah, especially with their children when they're so young. If they're regularly on antibiotics, yeah....'(P06)

'I imagine there would be some pockets of the community that it [antibiotic resistance] might be an issue for.' (P04)

'I think it's a big problem. People like to get antibiotics and just solve things instantly. Like people don't like to wait and see what happens, they like to get something-even if they think it's going to work or not, they just—something to make it better.' (P15)

Most participants described resistance as a problem that will not impact them individually-'I don't think it's a big issue for me' (P09); 'I think I'll get through my life without it impacting on it' (P21). A few participants described their worry about antibiotic resistance, although by many it was viewed as a future or a hypothetical concern:

'Oh, huge, I don't want that to happen... Um, well, if she got sick and constantly needed antibiotics... you know, then obviously in-as she gets older, they'd stop working as much as you wouldn't be able to treat infections as much and I don't want that to happen' (P03)

'... it could become a big problem if the so-called superbugs, um, come out and about later on, yeah.' (P09)

'It still concerns me, um, because someone as young as my two year-old son-I guess in an older person, it's perhaps not as concerning because over the course of a life time. but I think the message is out there that 
Table 1 Participant characteristics

\begin{tabular}{|c|c|c|c|c|}
\hline Participant ID & Participant age (years) & Gender & Presenting condition & If child patient, age (years) \\
\hline P01 & 18 & Female & Sore throat & \\
\hline P02 & 73 & Male & Acute bronchitis & \\
\hline P03 & 34 & Female & $\begin{array}{l}\text { Acute otitis media } \\
\text { (AOM) }\end{array}$ & 1 \\
\hline P04 & 47 & Female & Sore throat & \\
\hline P05 & 37 & Female & Sore throat & 1.3 \\
\hline P06 & 34 & Female & $\begin{array}{l}\text { Unspecified } \\
\text { acute respiratory } \\
\text { infection (ARI) }\end{array}$ & 11 \\
\hline P07 & 38 & Female & Acute bronchitis & \\
\hline P08 & 28 & Female & Acute bronchitis & \\
\hline P09 & 32 & Female & Acute bronchitis & 2 \\
\hline P10 & 22 & Male & Acute bronchitis & \\
\hline P11 & 27 & Female & Sore throat & \\
\hline P12 & 64 & Male & Acute bronchitis & \\
\hline P13 & 52 & Male & Acute bronchitis & 3 \\
\hline P14 & 39 & Male & Acute bronchitis & 2 \\
\hline P15 & 36 & Female & AOM & 6 \\
\hline P16 & 43 & Female & Acute bronchitis & 3 \\
\hline P17 & 18 & Female & Sore throat & \\
\hline P18 & 43 & Female & Sore throat & \\
\hline P19 & 70 & Female & Acute bronchitis & \\
\hline P20 & 45 & Female & Sore throat & \\
\hline P21 & 34 & Male & Acute bronchitis & \\
\hline P22 & 30 & Female & AOM & 4 \\
\hline P23 & 74 & Female & Acute bronchitis & \\
\hline P24 & 25 & Female & Acute bronchitis & 1.3 \\
\hline P25 & 24 & Female & Sore throat & \\
\hline P26 & 18 & Female & Acute bronchitis & \\
\hline P27 & 36 & Female & Unspecified ARI & 3 \\
\hline P28 & 21 & Male & Unspecified ARI & \\
\hline P29 & 50 & Female & Unspecified ARI & \\
\hline P30 & 34 & Female & Acute bronchitis & 2 \\
\hline P31 & 38 & Female & Acute bronchitis & 4.5 \\
\hline P32 & 35 & Female & AOM & 1.8 \\
\hline
\end{tabular}

maybe you need to think twice before (using antibiotics)' (P09)

Theme 3: poor awareness that resistance can spread between family members but concern that it can

Most participants did not know that antibiotic resistance can spread between people who are in close proximity, such as family members- No, I didn't even know it could spread' (P24). Some thought it would be possible:

'Um, I've never really thought about it before. My initial answer would be no, but I guess like if-yeah I guess if one of the children had a bug that was tougher, and they gave that to the other child, then, yeah, I guess, yeah, I guess it would be' (P32).

When participants were told by the interviewer that it can, the most common reaction was concern 'concerned. Yeah, it's not a good thing' (P14) and shock 'Oh, shocked. No, I never knew that.' (P01), with some insight into the significance of the problem 'So by one person using antibiotics can create problems for the whole family... Yeah. Well, that's, um, not real good, is it?' (P19)

Some participants suggested strategies to minimise the spread of resistance such as decreasing antibiotic use '.... 
so not using them too much' (P03) or with hand hygiene ('hand sanitiser' (P21), 'wash hands' (P07)).

Theme 4: low awareness that resistance can decay with time and variable impact of this knowledge on attitudes towards future antibiotic use

Most participants did not know that antibiotic resistance could decay over time:

'Oh, I've got no idea, I thought it just- that it stayed for a lifetime if you were resistant to it.' (P24)

'Oh, a long time. You'd have to-it'd take a lot of different ways to treat it' (P03)

'I imagine not, because once it's in your system, it remains there' (P09)

There was wide variation on estimation of the time to decay, ranging from days to decades:

'It wouldn't be; you wouldn't think within a couple of days... But I'm not saying 12 months or anything like that' (P19)

'Oh, probably ten years or something, crazy' (P15)

After explanation from the interviewer that antibiotic resistance does decay, some participants were more hopeful about the problem of antibiotic resistance:

'...it's promising to know that there is a chance ... given enough time, then they [Antibiotics] could work again' (P21)

'Yeah, well that's good that it could be then reversible' (P32)

'It makes me think that you could possibly go back to using those antibiotics if you had the similar problem maybe 18 months down the track' (P10)

It was assumed by some that science will come up with solutions to manage antibiotic resistance in the future:

'I don't think it will go away, but I think maybe people are coming up with different solutions to fight it rather than antibiotics or different ways of switching off you know our body's responses and things like that.' (P15)

'it will be interesting over the next $10-15$ years. I think that probably there'll be some really good break throughs in-in the engineering and the science behind antibiotics...' (P21)

The impact of knowing about resistance decay on attitude towards antibiotic use was variable. Some participants indicated no change ('No different than I said before. If it means it's (antibiotics) going to save my life and help me in my health, it wouldn't make any difference at all. (P23)), whereas others expressed that knowing this made them more cautious:

'That makes me really think about it-taking antibiotics only if you really need to' (P08)

'Especially for the children it would a lot scarier that they wouldn't be able to be treated ... if they were sick and something. It's quite frightening.' (P22)

Theme 5: antibiotics are perceived as sometimes necessary, with some awareness and consideration of their harms

Antibiotics were seen as beneficial by many participants ('only thing that helps' (P20)). The most commonly reported perceived benefits were decreased duration of illness ('taking antibiotics would make me better quicker' (P11) and decreased severity or progression of the infection ('to make sure it doesn't go to any further stages of infection.' (P06)).

Some participants believed in the need for antibiotics, despite being told by their GP that antibiotics would not help with viruses or provide better outcomes for them:

'...the doctor said oh it's a virus, I said well I'm going to be looking after my grandchildren, it's school holidays, and I needed something to help me get over this. ... and she said but they are not going to help you. I said well it's my decision at the time to have them because I didn't want my children to have what I had, you know. It was just a very bad virus I had, you know. But anyway, the antibiotics did work.' (P23)

Some participants were reluctant to take antibiotics for minor self-limiting illnesses, such as ARIs, and preferred to reserve antibiotic use for severe infections-'I would be hesitant. So, yes, maybe each time my doctor gives me antibiotics, I would ask is that necessary?' (P07), with some concerned about not wanting to overuse antibiotics-'should be more carefully applied and perhaps conservatively used.' (P18). Others' attitudes about antibiotic use were not influenced by illness severity-'... doesn't really change my opinion of it... certain antibiotics really work' (P25). Some participants' reasons for not using antibiotics were to 'give the body the best fighting chance' (P15) and by 'trying natural healing and staying healthy in the first place' (P13).

The few participants who had personal experience of antibiotic resistance were particularly cautious about antibiotic use:

'...because of my bronchitis... I have taken other medications that haven't worked. The-the doctors then had to change it... to a different medication. Yeah. Because I become resistant to others so I'm very fussy about taking them.' (P20)

There was great variability in participants' awareness of the potential harms of antibiotics. Many participants named potential side effects with commonly listed ones including 'vomiting', 'nausea', 'thrush' and 'diarrhoea'. Some mentioned 'possible resistance' as one of their concerns, but responses conveyed misunderstanding of what antibiotic resistance actually is. Some participants were not aware that antibiotics had potential harms'None that I'm aware of' (P21).

The patient-clinician relationship was viewed as very important when decisions about the management of 
infections were being made. Trust in the clinicians' recommendation for antibiotic use was mentioned by some-'as long as I can talk to my doctor and trust that the doctor is making the right decision' (P05).

Some participants described a lack of information and discussion with their clinician 'I don't have enough information to probably correctly make that call.' (P18) and were unaware of the option to not treat with antibiotics (that is, that the illness would get better without them) 'Um, well I guess when it's infected there's not really much other choice for that particular problem' (P32).

Some expressed a desire for more information about antibiotic resistance:

'Um, yeah, it would be good to know more about, um, how often you have to be taking them for resistance to build, whether individual, patient to patient' (P18).

... interested in knowing more information about (antibiotic resistance)' (P15)

\section{DISCUSSION}

This study has identified five major themes that related to people's understanding of antibiotic resistance and aspects of resistance such as resistance reversibility and spread among those in close proximity such as family or household members. While many participants articulated the link between antibiotic use and resistance, there was confusion about the nature of antibiotic resistance, which was often attributed to a trait of the body rather than bacteria in the microbiome. Many saw antibiotic resistance as a potential problem, rather than one that exists already, and that it was a consequence of and problem for the others in the community rather than them as an individual. Few appreciated the potential for antibiotic-resistant organisms to spread between those in close proximity, or that antibiotic resistance can decay.

Most participants reported the main benefit of antibiotic use was a decreased duration of illness. Some were aware of the potential for harm from antibiotics, including resistance. Some expressed reluctance to use antibiotics for minor self-limiting infections because of concern about overuse or misuse, whereas for others, it was not because of the potential harms but because of a preference for allowing their body to fight the infection naturally.

The poor understanding of the nature of antibiotic resistance has been found in previous studies in a general practice setting ${ }^{17}$ and in non-healthcare settings. ${ }^{18-20} \mathrm{~A}$ recent survey of the UK general adult population showed that lack of antibiotic resistance awareness was strongly associated with self-reported likelihood of requesting antibiotics for an influenza-like infection. ${ }^{21}$ It appeared that patients who had personal experience of antibiotic resistance were the most reluctant to use them again, preferring to reserve their use for serious illness. A survey of the general population in Germany found that people who knew of someone suffering from multidrug-resistant organisms, received more information by their clinician on antibiotic resistance and took less antibiotics for an infection (of any cause), compared with people who did not have any personal involvement. ${ }^{19}$

Our finding that the lack of individual 'ownership' of contribution to, or risk of, antibiotic resistance has previously been identified in a systematic review, ${ }^{12}$ which showed that the public do not believe they contribute to the development of antibiotic resistance. This is complemented by the finding that some participants believe that science will find a way to solve the resistance problem, which contradicts with messages about individuals needing to change their behaviour to minimise the problem.

Many public health campaigns convey the message of antibiotic resistance and how it is promoted by inappropriate antibiotic use and misuse. The effect of some campaigns has been analysed and a decrease in antibiotic use was found..$^{22}{ }^{23}$ Some of our findings might be useful in guiding and refining the content of messages in public health campaigns and clinical consultations about antibiotic resistance. For example, the information that developing antibiotic resistance in one's microbiome might also lead to resistance in people who are physically close to them, such as family members, could be an additional message in patient and public educational strategies to encourage appropriate antibiotic use. Most participants were quite concerned on learning about resistance spread and it prompted some to provide suggestions for how to minimise resistance development and its spreadsuggesting that perhaps this is the information that could contribute to altering people's attitudes and behaviour about antibiotic use for minor self-limiting illnesses.

Future research into the optimal information about antibiotic use and resistance to include in public messages and clinical consultations is recommended. This includes the potential utility of information about resistance decay and its impact on antibiotic use. Knowing that resistance decays over time if antibiotics are not used promoted hope in some people that the problem of resistance was not irreversible and that efforts to conserve antibiotic effectiveness by not using unless essential are worthwhile. However, for others, knowing that resistance decay occurs over time, may thwart attempts to encourage responsible antibiotic use.

At a clinical consultation level, better engagement with patients when antibiotics are being considered by providing a balanced discussion of antibiotic benefits and harms is encouraged. This conversation should include discussion that resistance is a potential harm of antibiotic use, and explanation of the possible consequences of it for the individual and the broader community.

A limitation of our study is that the sample is not representative of the wider Australian population as participants were recruited from one city in Australia, only those presenting with an ARI were invited, and the majority of participants were female. For a small number 
of participants (9), there is the risk that their knowledge about antibiotic resistance was influenced by their GPs' use of a patient decision aid-which included a very brief explanation of what resistance is, but not about the spread or decay of resistance. Although GPs who did not receive or use the aids may have mentioned resistance as part of the consultation regardless. Other limitations are that participants did not have the opportunity to provide feedback on the themes derived from the interviews and the short duration of the interviews-which could have affected the depth of the gathered information. Strengths of the study include the use of two researchers independently performing the thematic analysis and its contribution of new findings to this field. We are not aware of other studies which have explored people's knowledge about the potential for antibiotic-resistant organisms to spread between those who are in close proximity or that antibiotic resistance decays over time.

\section{CONCLUSION}

This study found that patients' understanding of many aspects of antibiotic resistance was poor including: what it is, individual contribution to its development, individual implications, its spread and decay. Incorporating messages that target misunderstandings into public health messages and clinical consultations may be an important strategy to encourage more appropriate use of antibiotics for illnesses such as ARIs.

Acknowledgements The authors would like to acknowledge and thank all patients and GPs who participated in this study.

Contributors MB, TH and CDM designed the study. MB recruited and interviewed participants. MB, EG, TH and CDM analysed the data. MB drafted the original manuscript and EG, TH and CDM contributed to writing and revising the manuscript. All authors read and approved the final manuscript.

Funding Funding for a PhD scholarship for the lead author was provided by the Australian National Health and Medical Research Council through a research grant for the Centre for Research Excellence in Minimising Antibiotic Resistance from Acute Respiratory Infections (\#1044904).

Disclaimer Centre for Research Excellence in Minimising Antibiotic Resistance from Acute Respiratory Infections had no role in study design, data collection, data analysis, data interpretation or writing of the report.

Competing interests None declared.

Patient consent for publication Not required.

Ethics approval Ethical approval was provided by the Human Research Ethics Committee at Bond University (\#0000015433).

Provenance and peer review Not commissioned; externally peer reviewed.

Data sharing statement № additional data are available.

Open access This is an open access article distributed in accordance with the Creative Commons Attribution Non Commercial (CC BY-NC 4.0) license, which permits others to distribute, remix, adapt, build upon this work non-commercially, and license their derivative works on different terms, provided the original work is properly cited, appropriate credit is given, any changes made indicated, and the use is non-commercial. See: http://creativecommons.org/licenses/by-nc/4.0/.

\section{REFERENCES}

1. Laxminarayan R, Duse A, Wattal C, et al. Antibiotic resistance-the need for global solutions. Lancet Infect Dis 2013;13:1057-98.

2. Bell BG, Schellevis F, Stobberingh E, et al. A systematic review and meta-analysis of the effects of antibiotic consumption on antibiotic resistance. BMC Infect Dis 2014;14:13.

3. Costelloe C, Metcalfe C, Lovering A, et al. Effect of antibiotic prescribing in primary care on antimicrobial resistance in individual patients: systematic review and meta-analysis. BMJ 2010;340:c2096.

4. Bakhit M, Hoffmann T, Scott AM, et al. Resistance decay in individuals after antibiotic exposure in primary care: a systematic review and meta-analysis. BMC Med 2018;16:126.

5. Pan $\mathrm{Y}$, Henderson J, Britt H. Antibiotic prescribing in Australian general practice: how has it changed from 1990-91 to 2002-03? Respir Med 2006;100:2004-11.

6. Spinks A, Glasziou PP, Del Mar CB. Antibiotics for sore throat. Cochrane Database Syst Rev 2013;11:CD000023.

7 Venekamp RP, Sanders SL, Glasziou PP, et al. Antibiotics for acute otitis media in children. Cochrane Database Syst Rev 2015;6:CD000219.

8. Smith SM, Fahey T, Smucny J, et al. Antibiotics for acute bronchitis. Cochrane Database Syst Rev 2017;6:CD000245.

9. Huttner B, Goossens H, Verheij T, et al. Characteristics and outcomes of public campaigns aimed at improving the use of antibiotics in outpatients in high-income countries. Lancet Infect Dis 2010;10:17-31.

10. Coxeter PD, Mar CD, Hoffmann TC. Parents' Expectations and Experiences of Antibiotics for Acute Respiratory Infections in Primary Care. Ann Fam Med 2017;15:149-54.

11. Cockburn J, Pit S. Prescribing behaviour in clinical practice: patients expectations and doctors' perceptions of patients' expectations--a questionnaire study. BMJ 1997;315:520-3.

12. McCullough AR, Parekh S, Rathbone J, et al. A systematic review of the public's knowledge and beliefs about antibiotic resistance. $J$ Antimicrob Chemother 2016;71:27-33.

13. Stewardson AJ, Vervoort J, Adriaenssens N, et al. Effect of outpatient antibiotics for urinary tract infections on antimicrobial resistance among commensal Enterobacteriaceae: a multinational prospective cohort study. Clin Microbiol Infect 2018;24:972-9.

14. Hoffmann TC, Del Mar CB. Effect of decision aids for acute respiratory infections on the use of antibiotics in general practice: a cluster randomised controlled trial. Trial ID no. ACTRN12616000644460. Australian New Zealand Clinical Trials Registry. 2016. https://www.anzctr.org.au/Trial/Registration/ TrialReview.aspx?id=370698\&isReview=true (Accessed Jan 2019).

15. Streubert HJ, Carpenter DR. Qualitative research in nursing: advancing the humanistic imperative. Philadelphia: Lippincott Williams \& Wilkins, 2011.

16. Braun V, Clarke V. Using thematic analysis in psychology. Qual Res Psychol 2006;3:77-101.

17. Brookes-Howell L, Elwyn G, Hood K, et al. 'The body gets used to them': patients' interpretations of antibiotic resistance and the implications for containment strategies. J Gen Intern Med 2012;27:766-72.

18. Lum EPM, Page K, Nissen L, et al. Australian consumer perspectives, attitudes and behaviours on antibiotic use and antibiotic resistance: a qualitative study with implications for public health policy and practice. BMC Public Health 2017;17:799.

19. Schneider S, Salm F, Schröder C, et al. [Antibiotic intake and resistance development - Knowledge, experience and behavior among the German general population]. Bundesgesundheitsblatt Gesundheitsforschung Gesundheitsschutz 2016;59:1162-70.

20. Business G. Wellcome Trust. London, England. Exploring the consumer perspective on antimicrobial resistance. 2015. https:// wellcome.ac.uk/sites/default/files/exploring-consumer-perspectiveon-antimicrobial-resistance-jun15.pdf (January 2019).

21. Roope LSJ, Tonkin-Crine S, Butler CC, et al. Reducing demand for antibiotic prescriptions: evidence from an online survey of the general public on the interaction between preferences, beliefs and information, United Kingdom, 2015. Euro Surveill 2018;23:1700424.

22. Sabuncu E, David J, Bernède-Bauduin C, et al. Significant reduction of antibiotic use in the community after a nationwide campaign in France, 2002-2007. PLoS Med 2009;6:e1000084.

23. Goossens $\mathrm{H}$, Coenen $\mathrm{S}$, Costers $\mathrm{M}$, et al. Achievements of the Belgian Antibiotic Policy Coordination Committee (BAPCOC). Euro Surveill 2008;13. 